Artículos 



\title{
VERIFICACIÓN DE LAS PROPIEDADES DE MORTEROS DE PEGA INDICADAS EN EL CÓDIGO SÍSMICO DE COSTA RICA 2010
}

\author{
Ing. Marcela Delgado Martínez \\ Ing. Alejandro Navas Carro, MSc.
}

\begin{abstract}
Resumen
En este artículo se verifican las proporciones para morteros de pega establecidas por el Código Sísmico de Costa Rica 2010 (CSCR-10). Con este fin se fabricaron 18 mezclas diferentes de mortero, que corresponden a las proporciones establecidas en el CSCR 10 utilizando tres arenas diferentes, una de río (Guápiles) y dos de tajo (Guacalillo y La Garita). Además se incluyeron dos proporciones adicionales que corresponden a la práctica común de las construcciones costarricenses.

A estos morteros se les realizaron pruebas para determinar sus principales propiedades: plasticidad, retención de agua y resistencia a la compresión simple a tres edades de falla distintas (3, 7 y 28 días), utilizando las normas establecidas por la American Society for Testing and Materials (ASTM).
\end{abstract}

Palabras Clave: mortero, mezclas, agregados, resistencia de materiales

\section{Summary}

This article verifies the proportioning specifications for mortar indicated in the 2010 edition of the Costa Rican Seismic Code (CSCR-10). For this purpose, 18 different mortar batches were prepared which correspond to the six proportions established in the CSCR 10 using three different kinds of aggregate: one river sand (Guápiles) and two quarry sands (Guacalillo and La Garita). Besides, two additional proportions corresponding to the common practice in Costa Rican constructions were included.

These mortars were tested in order to determine their main properties: plasticity, water retentivity, and compressive strength in three different test ages (3, 7, and 28 days), using the standard test methods designated by the American Society for Testing and Materials (ASTM).

Keywords: mortar, batches, aggregates, strength of materials

Aprobado: 13 de julio del 2011 • Recibido: 19 de agosto del 2010

\section{INTRODUCCIÓN}

El mortero de pega se compone de uno o más materiales cementantes, arena y agua en cantidad suficiente para producir una mezcla plástica y trabajable. Su función principal es adherir las unidades de mampostería de forma que actúen como un elemento integral que tenga las características de funcionalidad y desempeño deseadas. Además, actúa como un sello que impide la penetración del aire y el agua, impermeabilizando las paredes y protegiendo el acero de refuerzo. Sin embargo, a diferencia de los bloques de mampostería, el mortero de pega posee una gran variabilidad por ser elaborado en obra. A esto se suman las diferentes fuentes de origen de los agregados y la proporción en que estos se mezclan.

A pesar de que el mortero de pega constituye entre un $7 \%$ y un $9 \%$ del volumen de la mampostería (Navas, 1999), sus propiedades tienen gran repercusión en el sistema final. Estas pueden dividirse en propiedades del mortero fresco y propiedades del mortero endurecido. Las primeras, determinan la facilidad de elaboración del mortero y se relacionan con 
las propiedades de los elementos estructurales acabados, mientras que las propiedades del mortero endurecido ayudan a determinar el desempeño de la mampostería terminada. Las propiedades más importantes del mortero fresco son la consistencia (o plasticidad) y la retención de agua; la adherencia y la resistencia a la compresión son las características más relevantes del mortero endurecido.

\section{Consistencia o plasticidad}

La consistencia o plasticidad de un mortero está directamente relacionada con su trabajabilidad, por lo que un mortero más plástico es más fácil de manipular y colocar. Un mortero con una consistencia apropiada se esparce fácilmente y permite la adecuada colocación del bloque sin derramarse.

\section{Retención de agua}

Es la resistencia a la pérdida de agua de mezcla debido a la absorción de las unidades de mampostería y a la evaporación. Una adecuada retención de agua permite que el mortero se mantenga en estado plástico por más tiempo, lo cual mejora la trabajabilidad y por lo tanto la adherencia.

\section{Resistencia a la compresión}

La resistencia a la compresión del mortero de pega influye en la resistencia a la compresión del sistema completo de mampostería, propiedad que es utilizada como parámetro de control de calidad del sistema (Drysdale, Hamid y Baker, 1994).

La resistencia a la compresión del mortero depende básicamente de la cantidad de material cementante utilizado. No es recomendable usar una resistencia mayor a la requerida ya que disminuye la trabajabilidad y el aumento en la resistencia del mortero no es proporcional al aumento en la resistencia de la mampostería (Farny, Melander y Panarese, 2008).

La norma ASTM C 109 (2005) establece el procedimiento para determinar la resistencia a la compresión simple por medio de la falla de cubos de mortero de $50 \mathrm{~mm}$ de arista.

En cuanto al tema de la mampostería en Costa Rica, en los últimos años se han desarrollado una serie de investigaciones, dado que es uno de los

Cuadro 1. Proporciones por volumen establecidas en el CSCR-10.

\begin{tabular}{|c|c|c|c|c|c|}
\hline $\begin{array}{l}\text { Combinación de materiales } \\
\text { cementantes }\end{array}$ & $\begin{array}{l}\text { Tipo de } \\
\text { Mortero }\end{array}$ & Cemento & $\begin{array}{l}\text { Cemento de } \\
\text { mampostería }\end{array}$ & $\begin{array}{c}\text { Cal } \\
\text { hidratada }\end{array}$ & $\begin{array}{l}\text { Arena en condición } \\
\text { húmeda y suelta }\end{array}$ \\
\hline Cemento-cal hidratada & A & 1 & 0 & $1 / 4$ & 3 \\
\hline Cemento-cal hidratada & B & 1 & 0 & $1 / 2$ & 4 \\
\hline Cemento-cal hidratada & $\mathrm{C}$ & 1 & 0 & $3 / 4$ & 5 \\
\hline $\begin{array}{l}\text { Cemento-cemento de } \\
\text { mampostería }\end{array}$ & A & 1 & 1 & 0 & 5 \\
\hline $\begin{array}{l}\text { Cemento-cemento de } \\
\text { mampostería }\end{array}$ & B & $1 / 2$ & 1 & 0 & 4 \\
\hline $\begin{array}{l}\text { Cemento-cemento de } \\
\text { mampostería }\end{array}$ & $\mathrm{C}$ & 0 & 1 & 0 & 3 \\
\hline
\end{tabular}


métodos constructivos más utilizados en el país. Carballo (2004), Carvajal (2000), Cascante (2001) y Navas (1999) analizaron las propiedades de la mampostería utilizando materiales disponibles en el mercado nacional; variando para cada estudio las dimensiones de los bloques, el material con que están fabricados, la utilización de concreto de relleno y su calidad, las proporciones de mortero de pega, entre otros factores.

No obstante, en lo que respecta al estudio individual de los componentes de la mampostería, específicamente el mortero de pega, la información relacionada con las condiciones particulares del medio costarricense es más limitada. Rojas (1993) estudió el efecto que produce en los morteros la inclusión de aditivos adherentes y de larga vida, mientras que Muñoz (1995) se enfocó en analizar la influencia de la cal en la resistencia a compresión de los morteros. Sin embargo, estos dos trabajos son anteriores al CSCR-10 por lo que no se utilizan las proporciones expuestas en este. Araya (1998) analizó la resistencia de cubos de mortero con respecto a las proporciones del mismo y a su fabricación. En esta investigación las mezclas de mortero se tomaron de construcciones ubicadas en el Valle Central por lo que se presentó una gran variabilidad en los resultados debido a las diferentes prácticas constructivas.

Queda reflejado en la investigación de Araya (1998) la predilección que existe en el sector constructivo nacional por morteros fabricados únicamente con cemento y arena, sin utilizar cal o cementos de mampostería.

\section{PROGRAMA EXPERIMENTAL}

\section{Caracterización de los materiales:}

\section{Cementos}

Se utilizaron dos tipos de cemento: de uso general (UG) y de albañilería; además se usó cal hidratada con el propósito de cumplir con los requerimientos para la combinación de materiales cementantes que presenta el CSCR-10.

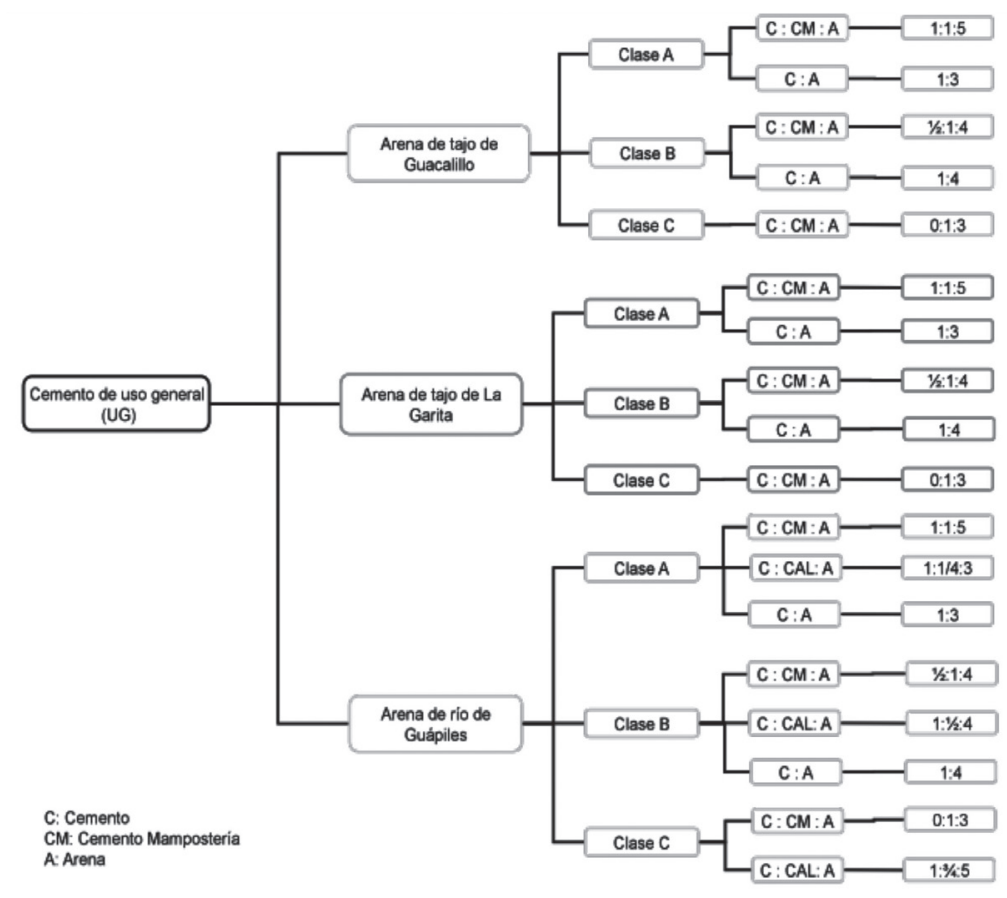

Figura 1. Proporciones utilizadas para la fabricación de los morteros de pega. 
Cuadro 2. Proporciones por volumen comúnmente usadas en construcciones costarricenses.

\begin{tabular}{cccc}
\hline Material cementante & Tipo de mortero & Cemento & $\begin{array}{c}\text { Arena en condición húmeda y } \\
\text { suelta }\end{array}$ \\
\hline Cemento & A & 1 & 3 \\
Cemento & B & 1 & 4 \\
\hline
\end{tabular}

Fuente: Araya (1998)

A estos materiales se les realizaron pruebas con el fin de determinar la resistencia a la compresión simple (ASTM C 190), el tiempo de fragua (ASTM C 191, 2004; ASTM C 266, 2008) y la gravedad específica (ASTM C 188, 2003).

\section{Agregado fino}

Las arenas se tamizaron por la malla $\mathrm{N}^{\circ}$ $4(4.75 \mathrm{~mm})$ con el fin de evitar partículas de tamaño inapropiado para la fabricación de morteros de pega de bloques. Se realizaron pruebas para determinar la granulometría (ASTM C 136, 2006), el porcentaje de material pasando la malla $\mathrm{N}^{\circ} 200$ por lavado (ASTM C 117, 2004), la absorción (ASTM C 128, 2004), la gravedad específica (ASTM C 128, 2004) y el densidad masiva de cada arena (ASTM C 29, 2003).

\section{Proporciones utilizadas}

Se utilizaron las proporciones establecidas en la sección A.1.4 del CSCR-10 (Cuadro 1).

Además se utilizaron las proporciones por volumen descritas en el Cuadro 2, las cuales constituyen la práctica común de las construcciones costarricenses (Araya, 1998).

\section{Fabricación del mortero}

Se realizaron 18 mezclas de mortero que corresponden a la combinación de las proporciones mostradas en los cuadros 1 y 2 con cada tipo de arena, tal como se muestra en la Figura 1.
Los morteros que contienen cal hidratada se fabricaron únicamente con arena de río, esto porque la variabilidad de la cal en el país es alta (Navas, 1999), por lo tanto no es apropiada como material de construcción; la realización de estos morteros es solamente una referencia general del comportamiento de las mezclas que contienen cal.

Los morteros se fabricaron utilizando la arena en condición húmeda y suelta, tal como lo especifica el CSCR-10 y se siguieron las normas ASTM C 109 (2005) y ASTM C 270(2006).

El agua utilizada en cada proporción es la necesaria para lograr un flujo entre $105 \%$ y $115 \%$, según lo indica ASTM C 109 (2005).

Las mezclas de mortero se realizaron en el Centro Tecnológico del Concreto (CETEC) de la compañía Holcim, en donde se determinó la plasticidad por medio de la prueba del Cono de Penetración (ASTM C 780, 2006) y se llevó a cabo el moldeo de cubos para la determinación de la resistencia a la compresión simple de especímenes de $5 \mathrm{~mm}$ de arista (ASTM C 109, 2005).

En el Laboratorio Nacional de Materiales y Modelos Estructurales (Lanamme UCR) se repitieron las mezclas con el fin de determinar la retención de agua de las mismas (ASTM C 1506, 2003).

\section{Pruebas realizadas a las mezclas de mortero}

\section{Prueba del cono de penetración (ASTM C 780)}

La prueba del cono de penetración o Vicat modificado permite determinar la consistencia 


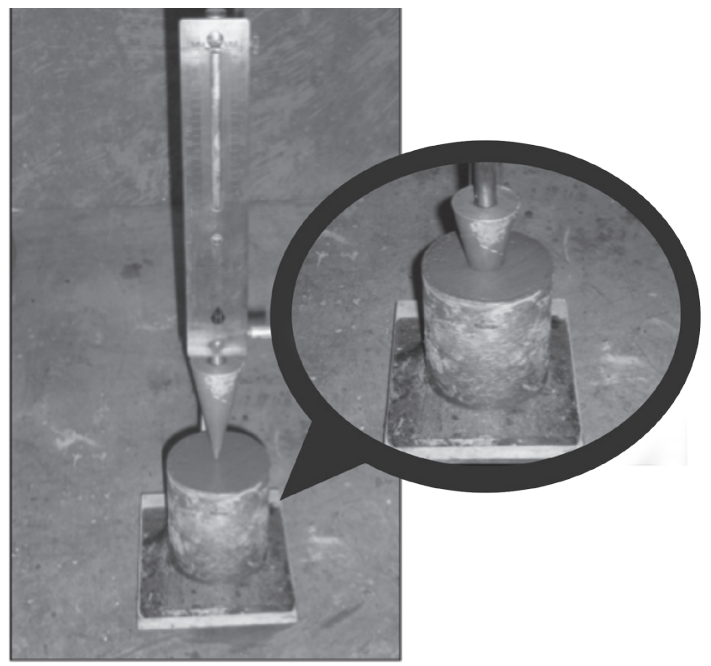

Figura 2. Prueba de Vicat modificado (ASTM C 780, 2006).

Fuente: Delgado (2007)

del mortero y se realiza una vez establecido un flujo adecuado. El equipo utilizado se muestra en la Figura 2 y consiste en una punta cónica que se deja caer sobre el mortero y cuya penetración es un indicador de la plasticidad de la mezcla. En la norma ASTM C 780 (2006) no se establece ningún rango de aceptación.

Debido a las características del equipo y al procedimiento del ensayo, esta es una prueba apta para realizarse en sitio.

\section{Prueba de retención de agua (ASTM C 1506)}

Al igual que en el ensayo de Vicat modificado, la retención de agua se mide después que se alcanzó el flujo deseado. Entonces la mezcla se coloca en el aparato mostrado en la Figura 3 y se le aplica una presión de succión de $7.0 \mathrm{kPa}$ $\pm 0.4 \mathrm{kPa}$. Terminado este proceso se vuelve a determinar el flujo y se calcula el porcentaje de retención de agua. Según ASTM C 270 (2006), el porcentaje de retención de agua mínimo debe ser $75 \%$.

Resistencia a la compresión simple (ASTM C 109)

Para la determinación de la resistencia a la compresión del mortero de pega se moldearon nueve especímenes de cada mezcla, de este modo fue posible fallar tres cubos a tres edades distintas (3, 7 y 28 días). El resultado de resistencia a los 28 días se comparó con los límites establecidos en el CSCR-10. En el Cuadro 3 se muestran estos límites.

\section{PRESENTACIÓN Y ANÁLISIS DE RESULTADOS}

\section{Caracterización de los materiales}

\section{Cementos}

El Cuadro 4 muestra una comparación de las características obtenidas para cada cemento Los valores obtenidos tanto para las gravedades específicas como para los tiempos de fragua de ambos tipos de cemento son similares. Sin embargo, existe una diferencia notable en las resistencias a compresión, siendo visiblemente mayor la resistencia de las muestras que contienen cemento de uso general. Esta situación se debe a que el cemento de mampostería busca aumentar la plasticidad, retención de agua y otras propiedades deseables en los morteros, incluso a expensas de la resistencia. 

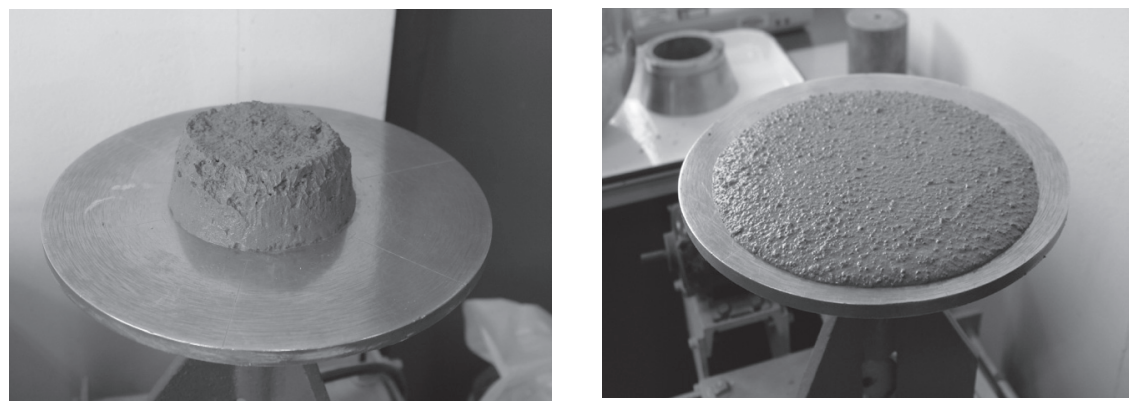

a) Medición del flujo antes de someter la mezcla a succión.
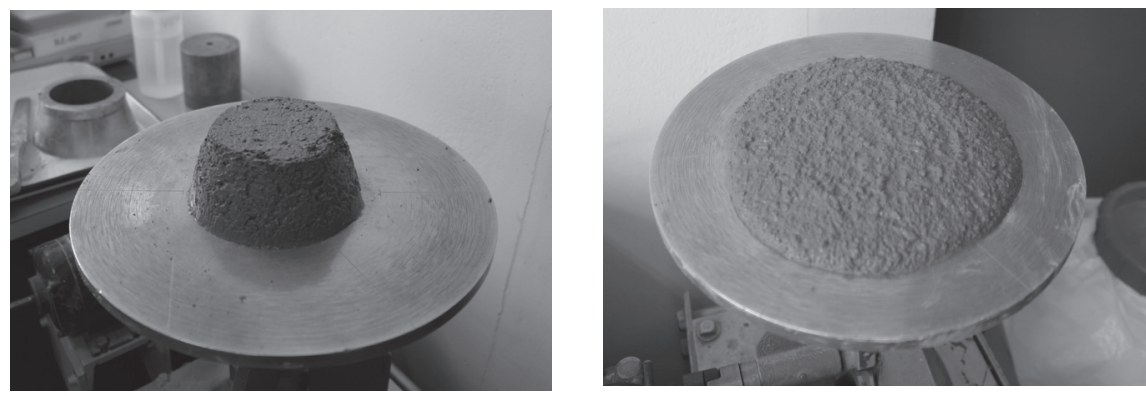

b) Medición del flujo después de someter la mezcla a succión.

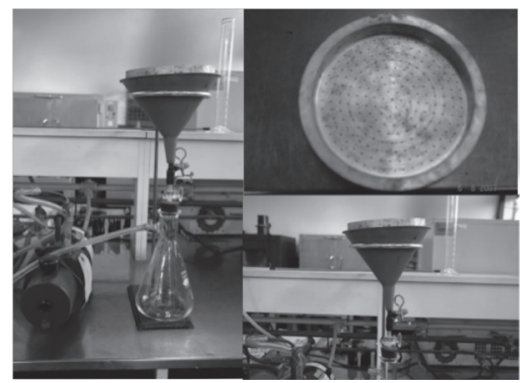

c) Equipo utilizado en la prueba.

Figura 3. Medición de la retención de agua de un mortero de pega.

Las características determinadas para ambos cementos cumplen con lo estipulado en la norma nacional de cementos hidráulicos, RTCR383:2004 (MEIC, 2005).

\section{Agregado fino}

El Cuadro 5 presenta las características de las arenas utilizadas para la fabricación de los morteros.
El cuadro 5 muestra diferencias entre los agregados de tajo con respecto al de río. En lo que respecta a la absorción, la arena de río de Guápiles tiene una absorción entre 4 y 5 veces menor que las arenas de tajo, lo que implica que es un agregado menos poroso. Esta situación se confirma en los valores de densidad masiva y las gravedades específicas, siendo siempre mayores las de Guápiles.

Otra característica importante que repercute en las mezclas de mortero es la cantidad de 
Cuadro 3. Resistencia a los 28 días para morteros de pega según CSCR-10.

\begin{tabular}{cc}
\hline Tipo de Mortero & Resistencia a los 28 días (MPa) \\
\hline A & 17.5 \\
B & 12.6 \\
C & 5.3 \\
\hline
\end{tabular}

Fuente: CPCSCR (2011)

Cuadro 4. Características de los cementos utilizados.

\begin{tabular}{cccc}
\hline & & $\begin{array}{c}\text { Cemento de Uso } \\
\text { General (UG) }\end{array}$ & $\begin{array}{c}\text { Cemento de } \\
\text { Albañilería }\end{array}$ \\
\hline \multicolumn{2}{c}{ Gravedad específica (ASTM C-188) } & 2.95 & 2.85 \\
Tiempo de Fragua & Inicial & 143 minutos & 126 minutos \\
(ASTM C-191 y ASTM C-266) & Final & 238 minutos & 260 minutos \\
Resistencia a la compresión simple & $\mathbf{3}$ días & $17.3 \mathrm{MPa}$ & $5.1 \mathrm{MPa}$ \\
(ASTM C-190) & $\mathbf{7}$ días & $25.6 \mathrm{MPa}$ & $9.9 \mathrm{MPa}$ \\
\hline
\end{tabular}

Fuente: Delgado (2007)

finos pasando la malla $\mathrm{N}^{\circ}$ 200. Se puede notar cómo los porcentajes para las arenas de tajo sobrepasan el 13\%, superando el límite establecido (10\%) por la ASTM C 144 (2004), mientras que para la arena de río se obtuvo solamente un $2 \%$.

\section{Proporciones utilizadas}

Las relaciones presentadas en el Cuadro 1 tienen como factor común la inclusión de un agente que mejora no sólo la trabajabilidad del mortero, sino su comportamiento en general. Este agente puede ser cal o cemento de mampostería.

A diferencia de estas proporciones, las que se muestran en el Cuadro 2 no cuentan con cal o cemento de mampostería y aún así son las comúnmente utilizadas en las construcciones costarricenses (Araya, 1998). Esta situación no es más que la consecuencia de las características de los materiales nacionales, ya que el cemento de mampostería no ha estado disponible regularmente en el mercado y la cal producida en el país es conocida por su variante calidad, lo cual no la hace apta para la construcción.

Las mezclas realizadas con cemento de mampostería o cal tienen una mayor plasticidad. Sumado a esto, la cantidad de finos de las arenas de tajo hacen que estos morteros sean más dóciles que los realizados con arena de río, para la cual las mezclas frecuentemente presentan una apariencia arenosa.

Por lo tanto, en lo que se refiere a plasticidad y facilidad de colocación, las proporciones que se incluyen en el CSCR-10 tienen ventaja sobre las expuestas en el Cuadro 2. Esta superioridad se refiere a morteros más uniformes y fáciles de colocar, lo que se traduce en mampostería de mejor calidad. 


\section{Relación agua-cemento}

La Figura 4 muestra las relaciones aguacemento $(\mathrm{w} / \mathrm{c})$ para las diferentes proporciones según el tipo de arena.

Las relaciones w/c para las diferentes combinaciones de proporciones y arenas se mantienen relativamente constantes, a excepción de la proporción 1:0:4, en la cual, la arena de tajo Guacalillo cuenta con una relación w/c, aproximadamente, un $20 \%$ menor que las otras dos arenas. Esta disminución es causada por la cantidad de finos presentes en dicha arena, lo que ocasiona que la mezcla sea más trabajable con menos cantidad de agua.

Es importante notar que las proporciones que cuentan con cemento de albañilería obtuvieron relaciones w/c menores que las que incluyen solamente cemento de uso general. El cemento de mampostería incluye aire a la mezcla, así como carbonato de calcio (calizas), por lo que es posible lograr mejores trabajabilidades en los morteros sin necesidad de agregar más agua.

La Figura 5 compara las relaciones w/c de los morteros fabricados con arena de río Guápiles, incluyendo las proporciones que utilizan cal 000.

A partir de la Figura anterior, se observa que el uso de cal también disminuye la relación w/c, pues tiene un efecto similar al del cemento de albañilería. Además, la cal permite que el mortero contenga el agua por más tiempo por lo que se conserva la trabajabilidad por un periodo mayor.

Otro parámetro importante en el cual influye la relación w/c es la resistencia del mortero. Teóricamente se conoce que entre mayor sea la proporción w/c de una mezcla, menor va a ser la resistencia alcanzada por esta. En la Figura 6, se muestra la relación entre la resistencia a la compresión simple y w/c según la proporción utilizada.

Al observar las mezclas realizadas con la misma proporción se refleja la tendencia antes mencionada, siendo más sensibles los morteros que contienen cemento de mampostería.

\section{Retención de agua}

La Figura 7 muestra cómo la retención de agua del mortero depende tanto de la proporción como del tipo de arena utilizado. Además se observa que la mayoría de las mezclas evaluadas están por debajo del $75 \%$ impuesto por ASTM C 1506 (2003).

Resulta importante notar que las proporciones cuyos valores de retención de agua eran menores al $50 \%$ son las de mayor uso en el país, por lo que los morteros fabricados con

Cuadro 5. Características de las arenas utilizadas.

\begin{tabular}{|c|c|c|c|}
\hline & \multicolumn{3}{|c|}{ Origen de la Arena } \\
\hline & Río de Guápiles & Tajo de Guacalillo & Tajo de La Garita \\
\hline Absorción & $3.8 \%$ & $14.5 \%$ & $23.3 \%$ \\
\hline Gravedad Específica, $\mathbf{G}_{\mathbf{S}}$ & 2.52 & 1.94 & 1.57 \\
\hline Gravedad específca saturado superficie seca, $\mathbf{G}_{\text {Sss }}$ & 2.61 & 2.22 & 1.94 \\
\hline Materiales pasando la malla $\mathrm{N}^{0} \mathbf{2 0 0}$ por lavado & $1.9 \%$ & $17.2 \%$ & $13.9 \%$ \\
\hline Peso unitario suelto, $\gamma_{\text {suelto }}(\mathrm{kg} / \mathrm{m} \square$ & 1636 & 1178 & 860 \\
\hline Peso unitario envarillado, $\gamma_{\text {envarillado }}(\mathrm{kg} / \mathrm{m} \square$ & 1713 & 1320 & 1010 \\
\hline
\end{tabular}


estas dosis, y particularmente los elaborados con arena de Guacalillo, son más susceptibles a sufrir problemas de contracción, agrietamiento, adherencia y permeabilidad.

Entre las tres arenas estudiadas la de tajo de La Garita es la que presenta un mejor comportamiento en cuanto a retención de agua, esto debido en algún grado a su alta absorción.

La Figura 8 muestra los valores de retención de agua para los morteros que contienen arena de río, esto incluye las proporciones que requieren cal. Es conocido que la cal ayuda a mejorar la retención de agua del mortero, sin embargo, por los resultados obtenidos, no se observa una contribución evidente. Lo anterior puede deberse a la calidad variante de la cal utilizada en el mercado nacional, lo cual, como ya se mencionó anteriormente, no la hace apta para su uso en construcción.

\section{Plasticidad}

La Figura 9 muestra un gráfico que relaciona la prueba de Vicat modificado con el ensayo de flujo. En este gráfico se puede observar que aunque no se sigue un comportamiento definido, sí es posible establecer un rango para los resultados del Vicat que asegure un flujo entre $105 \%$ y $115 \%$.

La obtención de límites para los valores del Vicat modificado significaría la posibilidad de realizar en campo una medida indirecta del flujo y así poder estandarizar las plasticidades de los morteros utilizados. Lo anterior ayudaría no sólo en la calidad del mortero sino que además facilitaría la pega de bloques.

Los rangos obtenidos se expresan en el Cuadro 6. Es importante mencionar que los datos para cada arena son limitados y, por lo tanto, es posible que los rangos establecidos no revelen el comportamiento real de cada agregado. Sin embargo, la presentación de los mismos muestra un panorama general del comportamiento de una arena con respecto a las demás.

En general, los resultados revelan que los morteros hechos con arena de La Garita son más plásticos, lo cual resultó evidente en las mezclas, pues visualmente se presentaron más uniformes que aquellas moldeadas con arena de Guápiles o de Guacalillo. La uniformidad en el mortero ayuda a la permeabilidad y adherencia del mismo pues permite una mejor colocación.

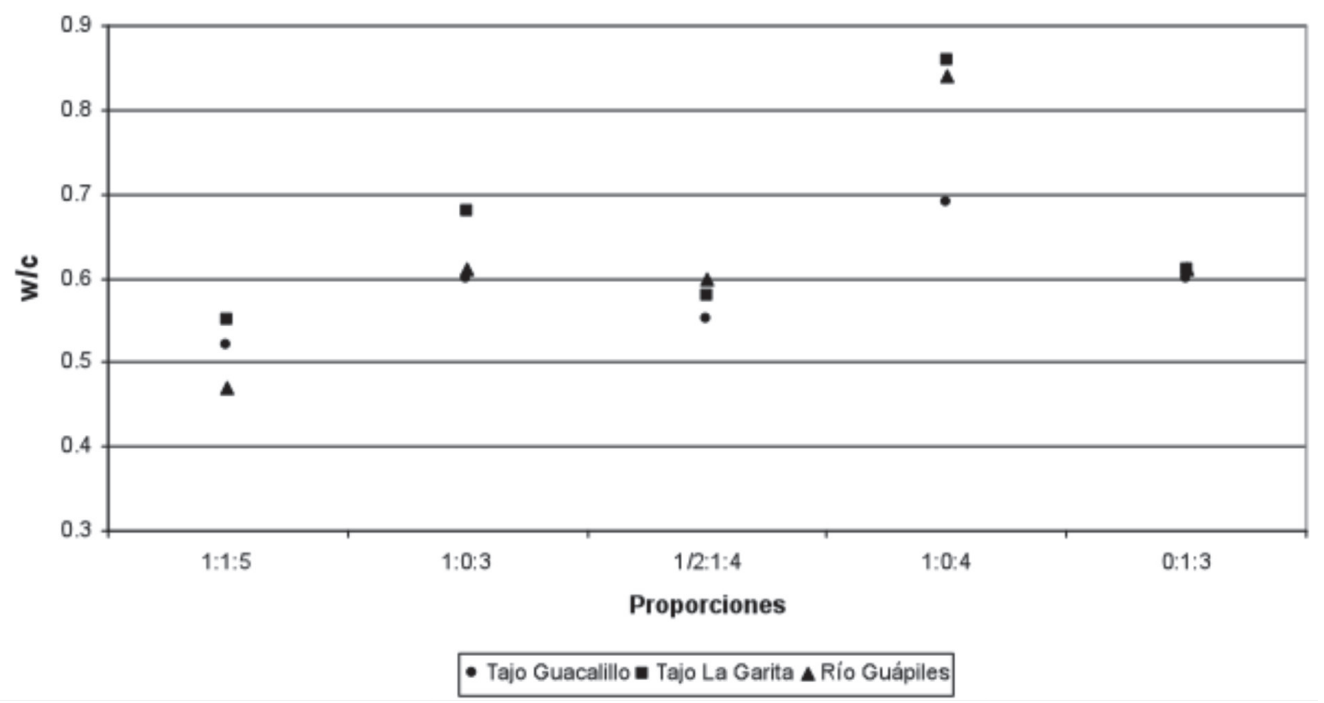

Figura 4. Relación agua-cemento para cada proporción según tipo de arena.

Fuente: Delgado (2007) 


\section{Resistencia a la compresión}

La resistencia a la compresión simple obtenida a los 28 días en cubos de mortero se muestra en la Figura 10.

Las resistencias, para los morteros fabricados con las proporciones propuestas en los cuadros 1 y 2, son superiores a los límites ya planteados con excepción de la proporción 1/2:1:4 con arena de río, la cual queda por debajo del estándar establecido para los morteros clase B mostrados en el cuadro 3 .

Las mezclas con arena de río obtuvieron resistencias superiores, para las proporciones con mayor cantidad de cementantes, sin embargo, para los morteros clase B y C las arenas de tajo tienen mayor resistencia. Esta tendencia se debe a la cantidad de finos de cada arena pues en estas mezclas, que contienen menos cementantes, los finos de la arena ayudan a rellenar espacios, los cuales en mezclas tipo A son sustituidos por cemento. Esto causa que las arenas con mayor porcentaje de finos logren resistencias más elevadas en estas proporciones.

La Figura 11 muestra las resistencias a 28 días de los morteros realizados con arena de río, aquí se incluyen las proporciones que contienen cal. Se puede observar que la inclusión de cal en la mezcla no afectó la resistencia del mortero de pega.

Además del valor de la resistencia a los 28 días, se debe considerar la relación entre la resistencia a la compresión y la edad de falla de los morteros. Esta se muestra en las Figuras 12 a 14.

Acerca de los morteros clase A se muestra en la Figura 12 cómo la mezcla fabricada con cal alcanza una resistencia mayor que el resto de las proporciones. En dicho gráfico se observa que el mortero clase A con cal obtuvo igual resultado que la proporción 1:1:5, ambas con arena de río. Esto se debe a que las mezclas clase A cuentan con una cantidad mayor de material cementante, por lo que no se ven afectadas con el uso de una arena más gruesa como lo es la arena de río.

Para los morteros clase B y C, mostrados en las Figuras 13 y 14 respectivamente, las mezclas con arena de río que contienen cal superan en resistencia a los morteros con el mismo agregado y que no la contienen. En estos casos, la cal ayuda a completar los espacios, lo que se traduce en mayores resistencias.

\section{CONCLUSIONES}

\section{Consistencia}

Las proporciones que contienen cal o cemento de mampostería resultan en morteros más plásticos y por lo tanto son mezclas que requieren menos agua para alcanzar trabajabilidades aceptables.

Los morteros fabricados con arena de tajo de La Garita son más plásticos. Las mezclas

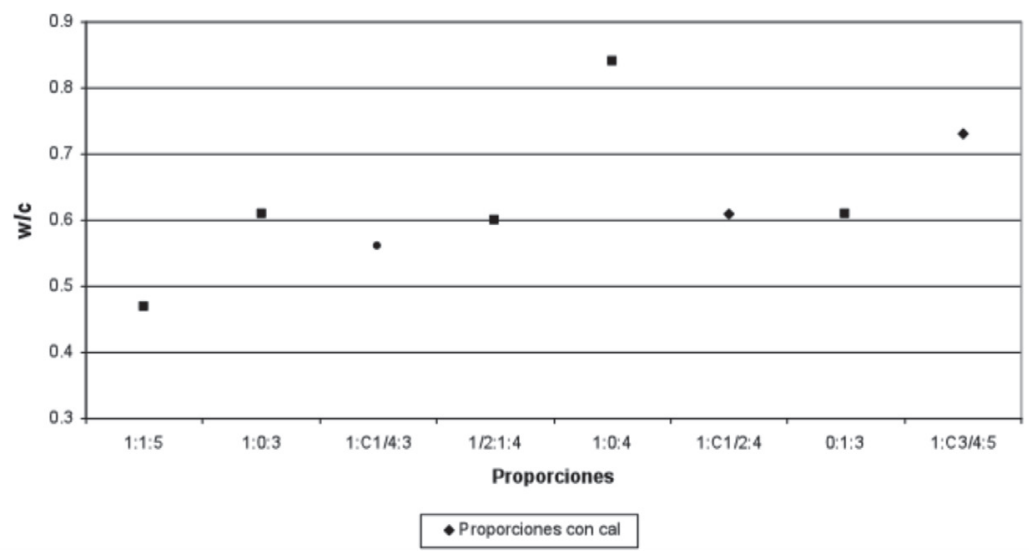

Figura 5. Relación agua-cemento para morteros realizados con arena de río de Guápiles. Fuente: Delgado (2007) 


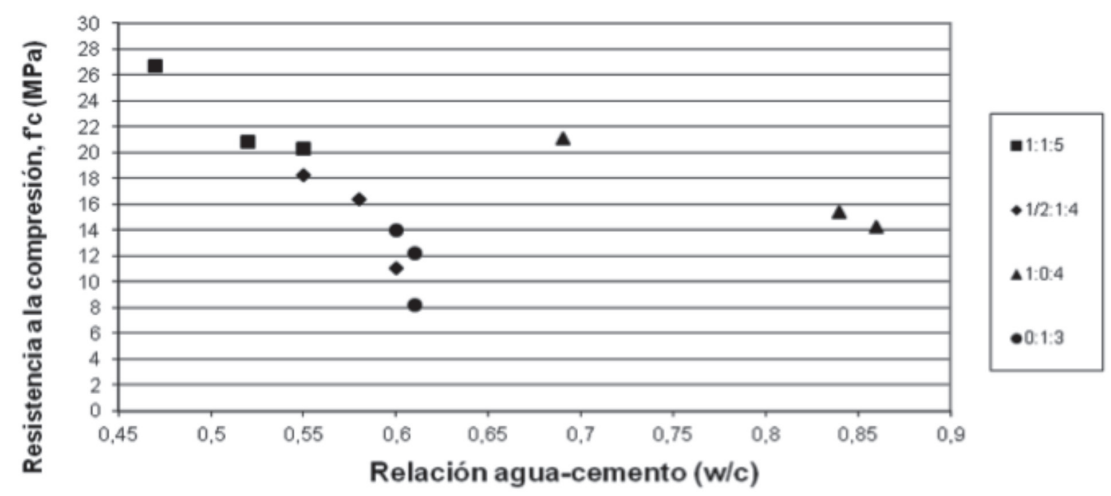

Figura 6. Relación entre w/c y resistencia a la compresión simple (f' $\mathrm{c}$ ) según la proporción utilizada.

Fuente: Delgado (2007)

Cuadro 6. Rangos para los valores del Vicat modificado correspondientes a un flujo de $110 \% \pm 5 \%$ para cada tipo de arena.

\begin{tabular}{ccccc}
\hline Tipo de arena & $\begin{array}{c}\text { Promedio } \\
(\mathbf{m m})\end{array}$ & $\begin{array}{c}\text { Desviación } \\
\text { estándar } \mathbf{S}(\mathbf{m m})\end{array}$ & $\begin{array}{c}\text { Rango 1 } \\
\text { Rango 2 }\end{array}$ \\
\hline Río de Guápiles & 30 & 4 & $\begin{array}{c}\text { Promedio } \pm \mathbf{1} \text { Desviación } \\
\text { estándar) }\end{array}$ & $\begin{array}{c}\text { (Promedio } \pm \text { 2 Desviación } \\
\text { estándar) }\end{array}$ \\
Tajo de Guacalillo & 28 & 4 & 26 a $34 \mathrm{~mm}$ & 22 a $38 \mathrm{~mm}$ \\
Tajo de La Garita & 38 & 7 & 24 a $32 \mathrm{~mm}$ & 20 a $36 \mathrm{~mm}$ \\
\hline
\end{tabular}

Fuente: Delgado (2007)

son homogéneas, lo cual hace que sean más fáciles de manipular. Esta situación agiliza la pega de bloques.

\section{Retención de agua}

El porcentaje de retención de agua es independiente de la proporción cuando se utilizan arenas de La Garita y de Guápiles.

Los morteros fabricados con agregado de Guacalillo y con proporciones sin cemento de mampostería tienen deficiencias de retención de agua, con porcentajes menores al 50\%. Estos son morteros más susceptibles a problemas de contracción, agrietamiento, adherencia y permeabilidad.

El agregado de La Garita es el que cuenta con un mejor desempeño, sin embargo el problema de retención de agua es generalizado en todas las mezclas.

El uso de cal en las proporciones no mejoró el porcentaje de retención de agua de las mezclas fabricadas en este trabajo.

Las deficiencias en cuanto a retención de agua son un problema de agregados y no de proporciones. 


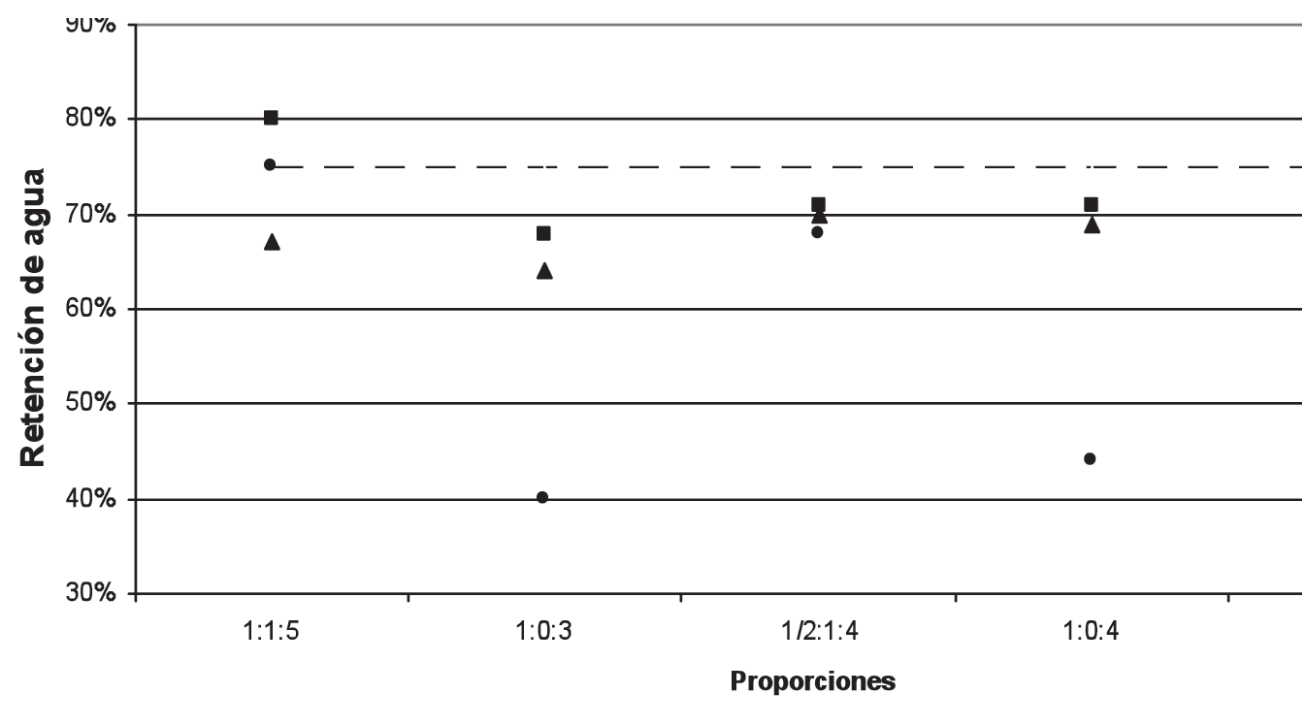

- Tajo Guacalillo Tajo La Garita \ Río Guápiles

Figura 7. Retención de agua para cada proporción según el tipo de arena.

Fuente: Delgado (2007)

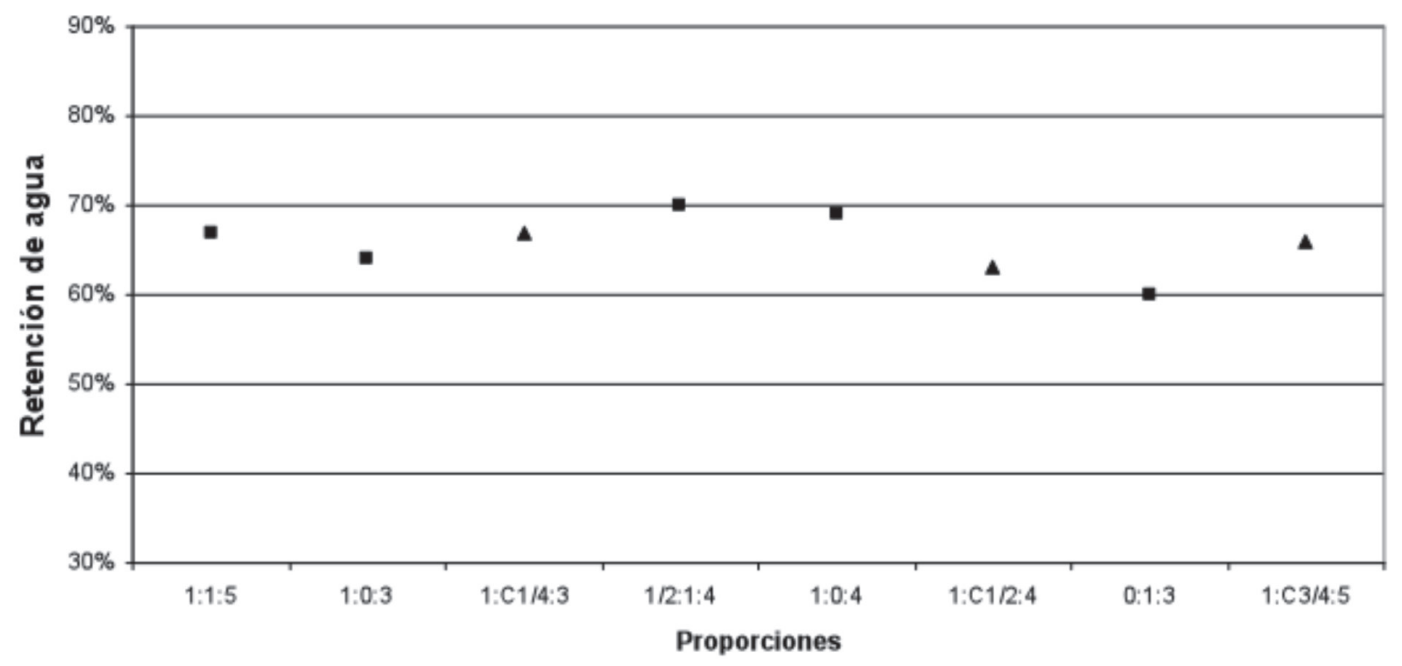

$\Delta$ Proporciones con Cal

Figura 8. Retención de agua para los morteros fabricados con arena de río de Guápiles.

Fuente: Delgado (2007) 


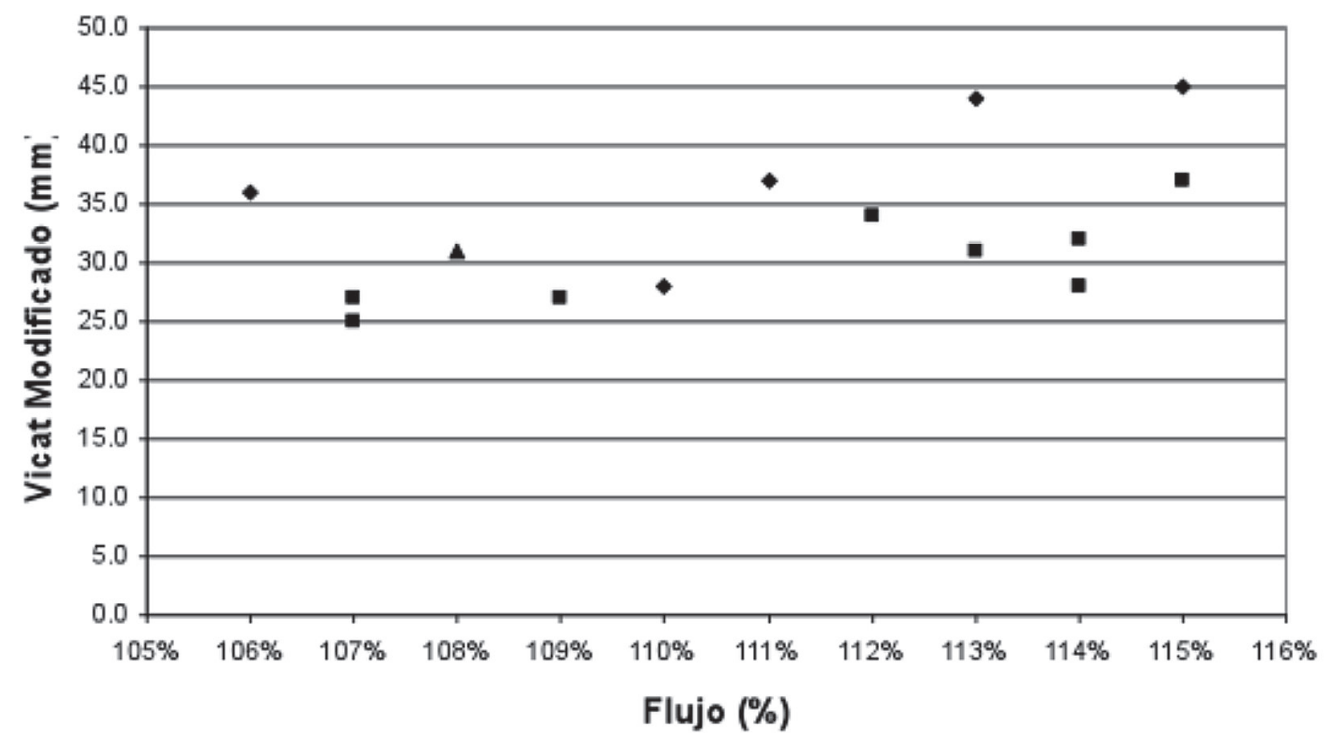

A Tajo Guacalillo • Tajo La Garita n Río Guápiles

Figura 9. Relación del flujo con la prueba de Vicat modificado.

Fuente: Delgado (2007)

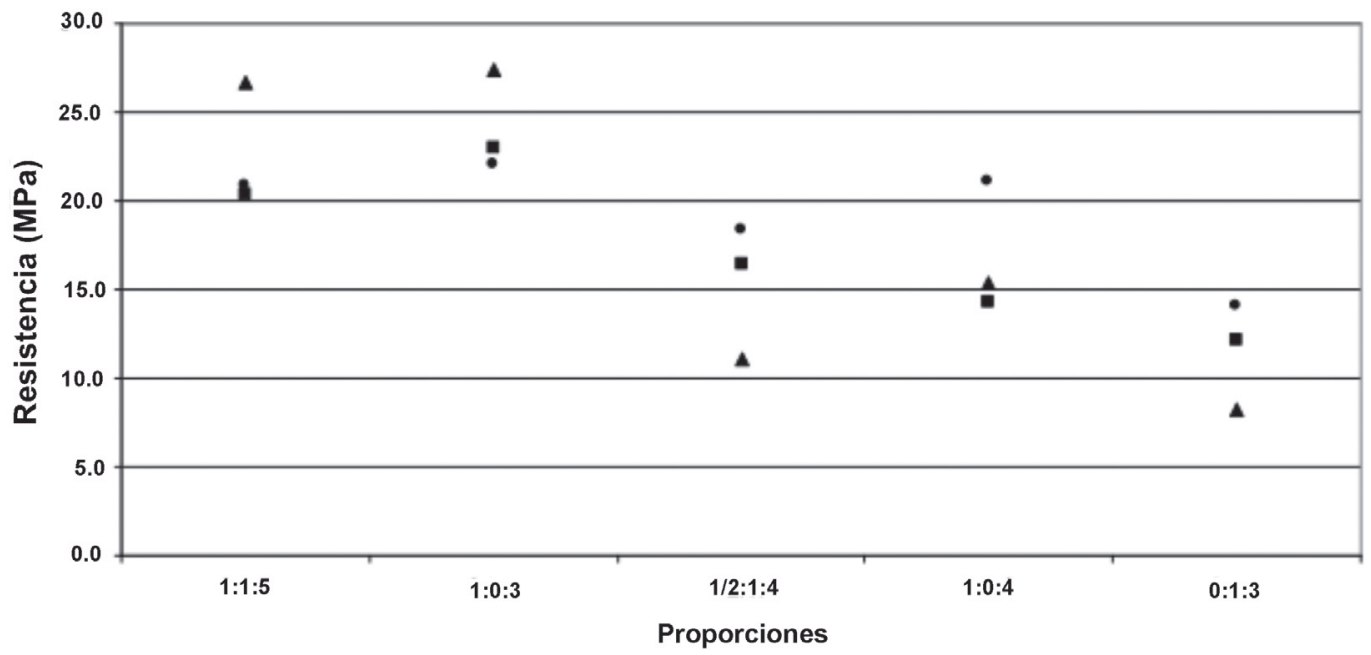

- Tajo Guacalillo a Tajo La Garita ^ Río Guápiles

Figura 10. Resistencia a los 28 días de morteros de pega para diferentes proporciones, según el tipo de arena.

Fuente: Delgado (2007) 


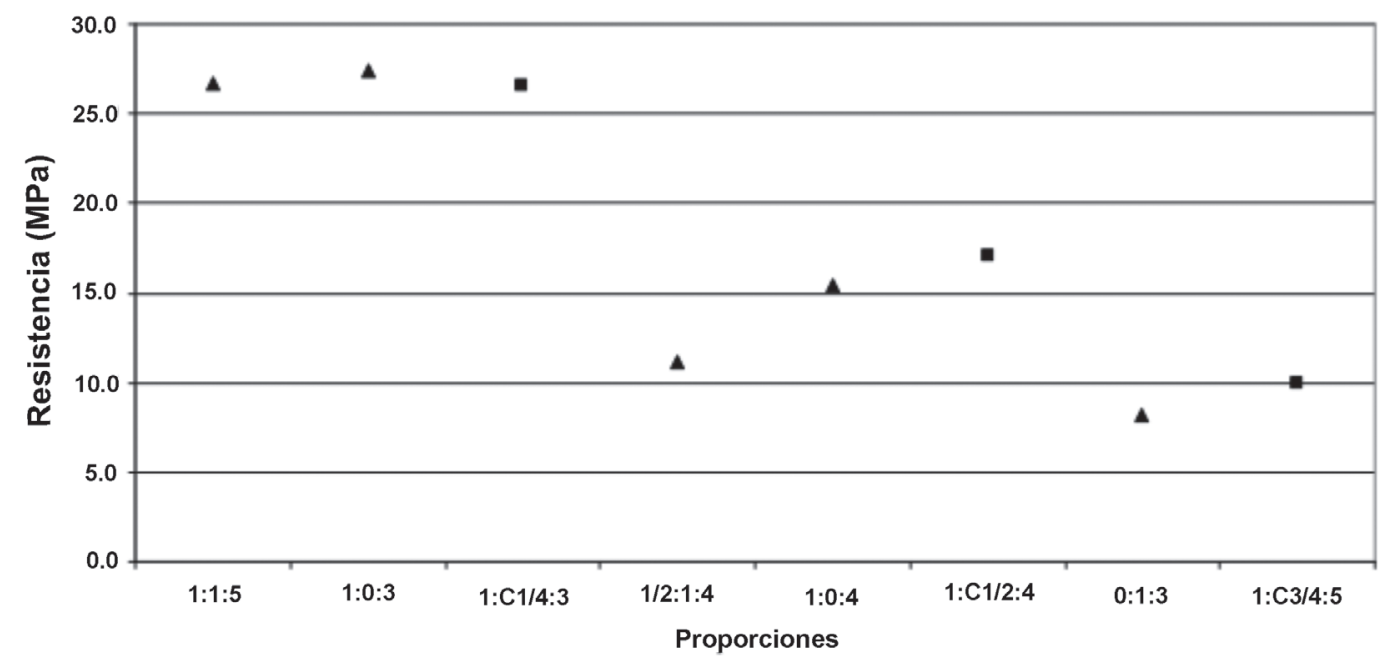

- Proporciones con cal

Figura 11. Resistencia a la compresión simple de morteros fabricados con arena de río Guápiles.

Fuente: Delgado (2007)

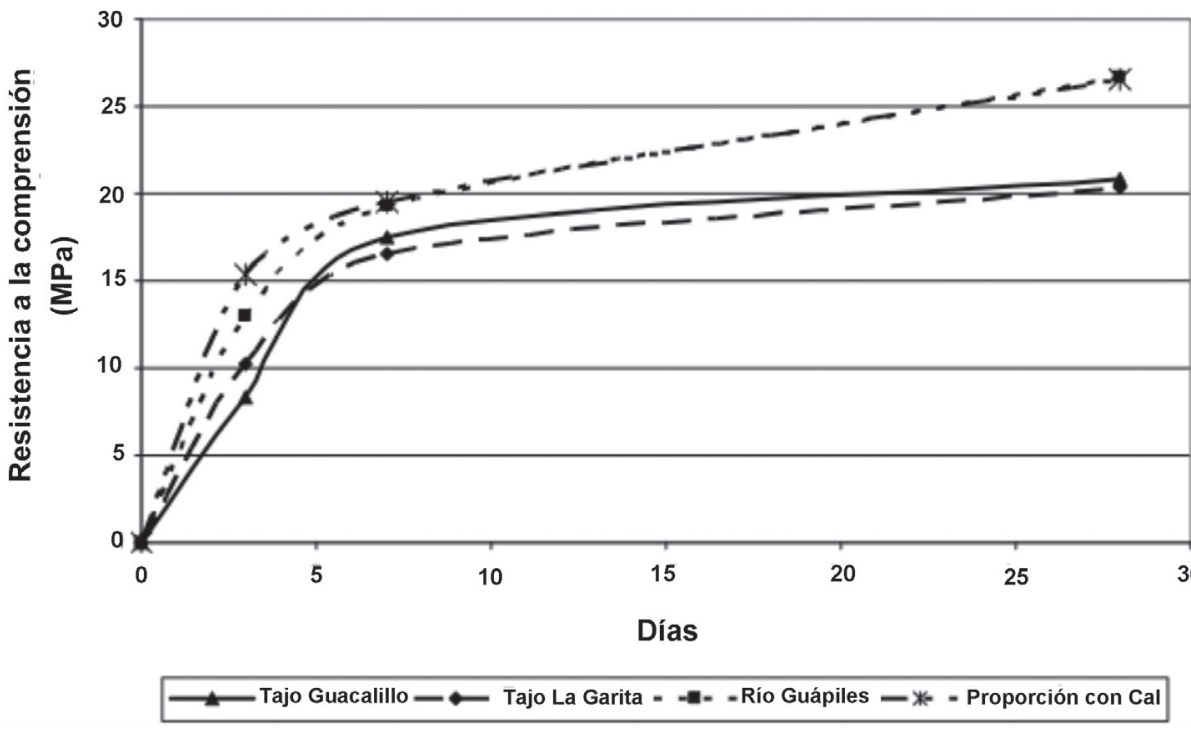

Figura 12. Relación entre la resistencia a la compresión y edad de falla para morteros clase A.

Fuente: Delgado (2007) 


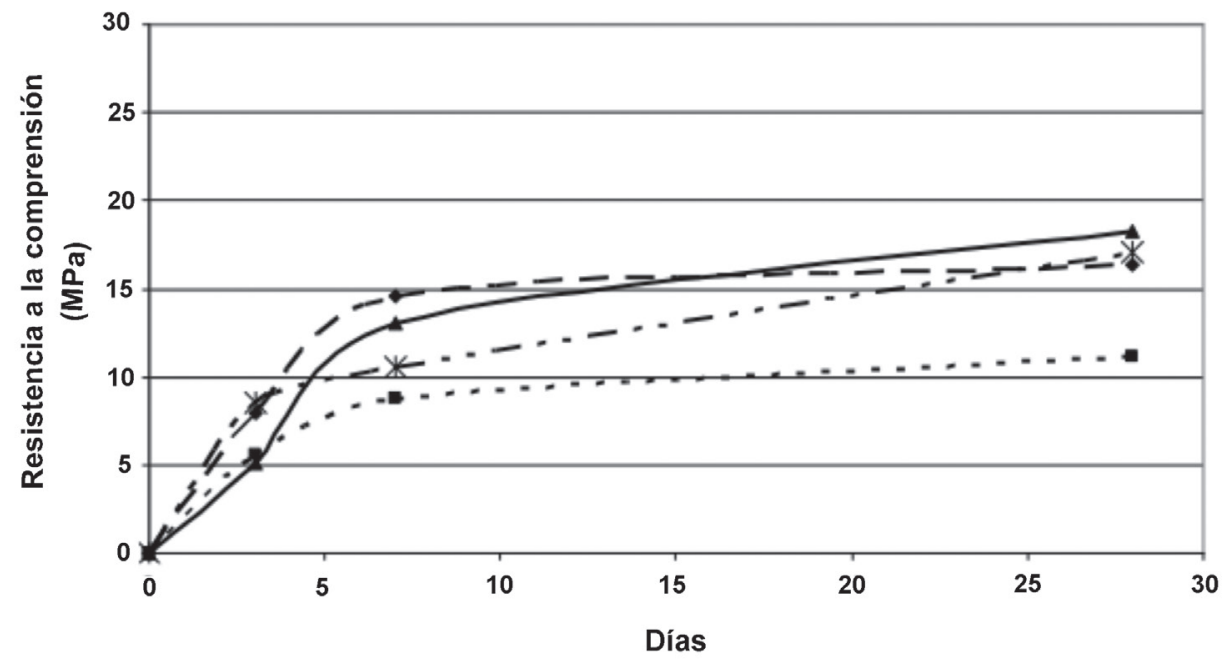

$\longrightarrow$ Tajo Guacalillo —— Tajo La Garita - -m- -Río Guápiles —

Figura 13. Relación entre la resistencia a la compresión y la edad de falla para morteros clase B. Fuente: Delgado (2007)

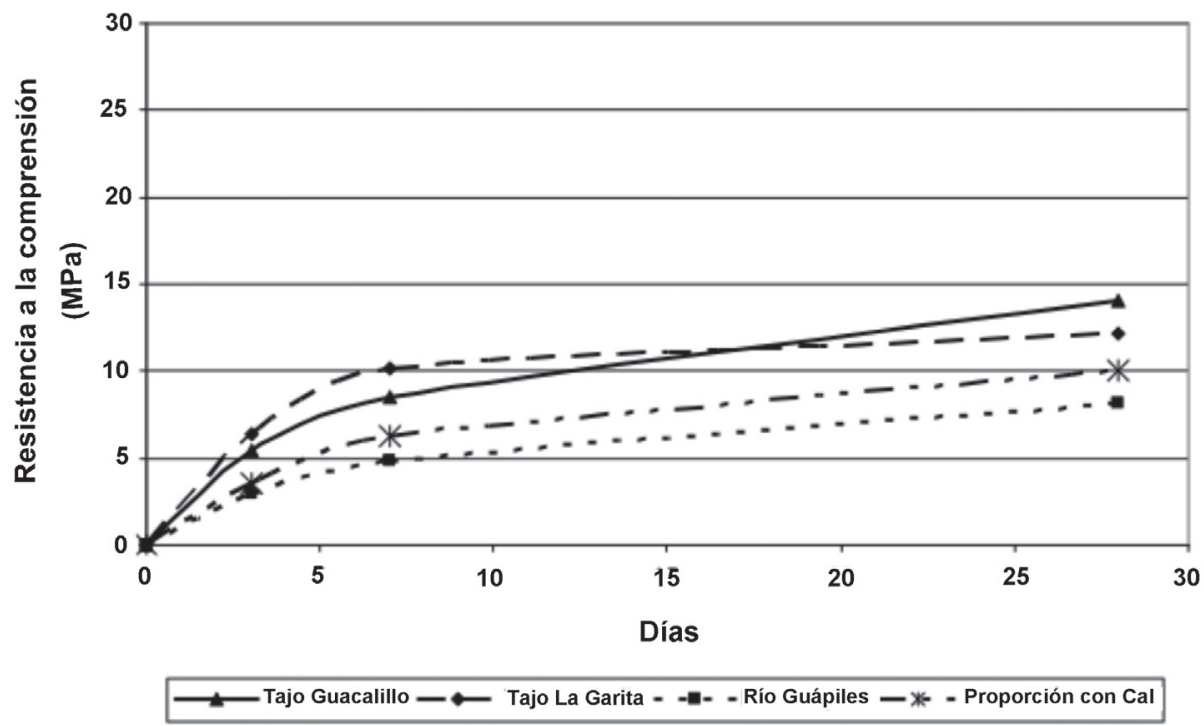

Figura 14. Relación entre la resistencia a la compresión y la edad de falla para morteros clase C. Fuente: Delgado (2007) 


\section{Resistencia a la compresión}

Las mezclas cumplieron con los estándares de resistencia a la compresión impuestos por ASTM C 270 (2006), con excepción de la proporción 1/2:1:4 con arena de río de Guápiles.

Los morteros clase A alcanzan resistencias más altas cuando se utiliza arena de río, mientras que las clases $\mathrm{B}$ y $\mathrm{C}$ tienen mejores resultados al utilizar arenas de tajo.

Las proporciones que utilizan cal presentan mejores comportamientos en cuanto a resistencia en morteros fabricados con arena de río.

\section{Relación entre la resistencia y la edad de falla}

Las mezclas que contienen agregado de río, para morteros clase $\mathrm{A}$, cuentan con resistencias mayores en todas las edades de falla. Los morteros fabricados con las arenas de tajo tienen una ganancia similar de resistencia.

El agregado de río, en el caso de los morteros clase $\mathrm{B}$ y $\mathrm{C}$, presenta una resistencia menor en todas las edades de falla. Con respecto a las mezclas con arenas de tajo, los morteros de La Garita alcanzan mayores resistencias tempranas, sin embargo, la tasa de crecimiento del agregado de Guacalillo después de los 7 días es mayor, por lo que a los 28 días aventaja en resistencia $\mathrm{La}$ Garita.

En términos generales, las proporciones indicadas en la Sección A.1.4 del CSCR 2010 cumplen con los requerimientos establecidos para resistencia y plasticidad, sin embargo, en lo que respecta a la retención de agua se muestran levemente por debajo del límite de aceptación. La deficiencia en cuanto a la retención de agua refleja más un problema de agregados y no de dosificaciones por lo que las proporciones planteadas en el CSCR-10 producen morteros con propiedades adecuadas en cuanto a resistencia y trabajabilidad.

\section{REFERENCIAS BIBLIOGRÁFICAS}

American Society for Testing and Materials, ASTM C 29. (2003). Standard Test Method for Bulk Density (Unit Weight) and Voids in Aggregate. Pennsylvania: ASTM.
American Society for Testing and Materials, ASTM C109. (2005). Standard Test Method for Compressive Strength of Hydraulic Cement Mortars (Using 2-in. or [50-mm] Cube Specimens). Pennsylvania: ASTM.

American Society for Testing and Materials, ASTM C117. (2004). Standard Test Method forMaterials Finer tha 75-x03BC;(NO200) Sieve in Mineral Aggregates by Washing. Pennsylvania: ASTM

American Society for Testing and Materials, ASTM C128. (2004). Standard Test Method for Density, Relative Density (Specific Gravity), and Absorption of Fine Aggregate. Pennsylvania: ASTM.

American Society for Testing and Materials, ASTM, C136. (2006). Standard Test Method for Sieve Analysis of Fine and Coarse Aggregates. Pennsylvania: ASTM

American Society for Testing and Materials, ASTM C144. (2004). Standard Specification for Aggregate for Masonry Mortar. Pennsylvania: ASTM.

American Society for Testing and Materials, ASTM C188. (2003). Standard Test Method for Density of Hydraulic Cement. Pennsylvania: ASTM.

American Society for Testing and Materials, ASTM C191. (2004). Standard Test Method for Time of Setting of Hydraulic Cement by Vicat Needle. Pennsylvania: ASTM.

American Society for Testing and Materials, ASTM C266. (2008). Standard Test Method for Time of Setting of Hydraulic Cement Paste by Guillmore Needles. Pennsylvania: ASTM

American Society for Testing and Materials, ASTM C270. (2006). Standard Specification for Mortar for Unit Masonry. Pennsylvania: ASTM.

American Society for Testing and Materials,ASTM C780. (2006). Standard Test Method for Preconstruction and Construction Evaluation of Mortars for Plain and Reinforced Unit Masonry. Pennsylvania: ASTM.

American Society for Testing and Materials, ASTM C1506.(2003). Standard Test Method for Water Retention of Hydraulic CementBased Mortars and Plasters. Pennsylvania: ASTM. 
Araya, M. (1998). Control de Calidad del concreto Estructural y del Mortero de Pega en Viviendas. Informe de Proyecto Final de Graduación para optar por el grado de Licenciatura en Ingeniería Civil, Escuela de Ingeniería Civil, Universidad de Costa Rica, San José, Costa Rica.

Carballo, G. (2004). Propiedades Mecánicas de la Mampostería con Bloques Sólidos de Arcilla. Informe de Proyecto Final de Graduación para optar por el grado de Licenciatura en Ingeniería Civil, Escuela de Ingeniería Civil. San José: Universidad de Costa Rica.

Carvajal, W. (2000). Propiedades Mecánicas de la Mampostería de Bloques Rellenos de Concreto de $12 \times 20 \times 40 \mathrm{~cm}$. Informe de Proyecto Final de Graduación para optar por el grado de Licenciatura en Ingeniería Civil, Escuela de Ingeniería Civil. San José: Universidad de Costa Rica.

Cascante, L. (2001). Propiedades Mecánicas de la Mampostería de Bloques Rellenos de Concreto de 20x20x40 cm. Informe de Proyecto Final de Graduación para optar por el grado de Licenciatura en Ingeniería Civil, Escuela de Ingeniería Civil. San José: Universidad de Costa Rica.

Comisión Permanente de Estudio y Revisión del Código Sísmico de Costa Rica (CPCSCR). (2011). Código Sísmico de Costa Rica 2010. Cartago: Editorial Tecnológica de Costa Rica.

Delgado, M. (2007). Verificación de las Proporciones para Morteros indicadas en el Código Sísmico de Costa Rica 2002. Informe de Proyecto Final de Graduación para optar por el grado de Licenciatura en Ingeniería Civil, Escuela de Ingeniería Civil. San José: Universidad de Costa Rica.

Drysdale, R., Hamid, A., Baker, L. (1994). Masonry Structures, Behavior and Design. New Jersey: Prentice Hall.

Farny, J., Melander, J. y Panarese, W. (2008). Concrete Masonry Handbook. Illinois: PCA.
Ministerio de Economía, Industria y Comercio, MEIC 32253. (2005). Reglamento técnico RTCR 383:2004 Cementos hidráulicos, especificaciones. San José: Imprenta Nacional.

Muñoz, J. (1995). Influencia de la Cal en la Resistencia a Compresión de Morteros. Informe de Proyecto Final de Graduación para optar por el grado de Licenciatura en Ingeniería Civil, Escuela de Ingeniería Civil. San José: Universidad de Costa Rica.

Navas, A. (1999). Propiedades Mecánicas de la Mampostería de Concreto. Proyecto de tesis para optar por el grado de Maestría en Ingeniería Civil, Escuela de Ingeniería Civil. San José: Universidad de Costa Rica.

Rojas,A.(1993). Evaluación del Comportamiento del Mortero de Albañilería, con Adherente y Larga Vida. Informe de Proyecto Final de Graduación para optar por el grado de Licenciatura en Ingeniería Civil, Escuela de Ingeniería Civil. San José: Universidad de Costa Rica.

\section{SOBRE EL AUTOR Y LA AUTORA}

\section{Alejandro Navas Carro}

Ingeniero Civil. Profesor de la Escuela de Ingeniería Civil, Universidad de Costa Rica

Director del Laboratorio Nacional de Materiales y Modelos Estructurales (LanammeUCR) de la Universidad de costa Rica. Apartado Postal: 2060, UCR, San José Costa Rica.

Teléfono: 2511 2508, Facsímil:2511 4440

Correo electrónico: alejandro.navas@ucr.ac.cr

\section{Marcela Delgado Martínez}

Ingeniera Civil. Funcionaria de Holcim Costa Rica S.A., Ingeniería

Teléfono: 2205-2700

Correo electrónico: marcela.delgadom@ @olcim.com 
